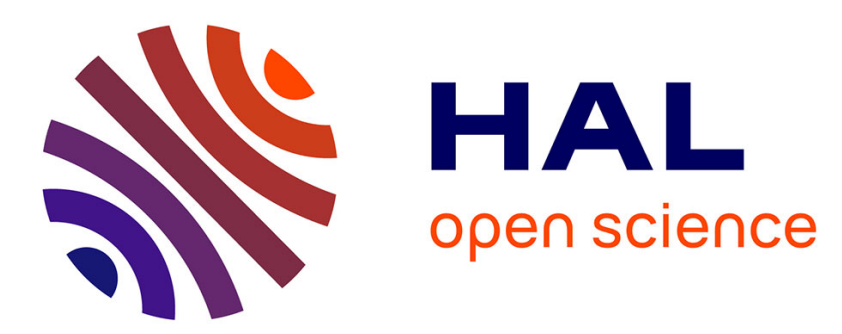

\title{
Robust controlled invariance for monotone systems: application to ventilation regulation in buildings
}

Pierre-Jean Meyer, Antoine Girard, Emmanuel Witrant

\section{To cite this version:}

Pierre-Jean Meyer, Antoine Girard, Emmanuel Witrant. Robust controlled invariance for monotone systems: application to ventilation regulation in buildings. Automatica, 2016, 70, pp.14-20. 10.1016/j.automatica.2016.03.004 . hal-01276261

\section{HAL Id: hal-01276261 \\ https://hal.science/hal-01276261}

Submitted on 19 Feb 2016

HAL is a multi-disciplinary open access archive for the deposit and dissemination of scientific research documents, whether they are published or not. The documents may come from teaching and research institutions in France or abroad, or from public or private research centers.
L'archive ouverte pluridisciplinaire HAL, est destinée au dépôt et à la diffusion de documents scientifiques de niveau recherche, publiés ou non, émanant des établissements d'enseignement et de recherche français ou étrangers, des laboratoires publics ou privés. 


\title{
Robust controlled invariance for monotone systems: application to ventilation regulation in buildings *
}

\author{
Pierre-Jean Meyer ${ }^{\mathrm{a}}$, Antoine Girard ${ }^{\mathrm{b}}$, Emmanuel Witrant ${ }^{\mathrm{c}}$ \\ ${ }^{a}$ KTH Royal Institute of Technology, Department of Automatic Control, 10044 Stockholm, Sweden \\ ${ }^{\mathrm{b}}$ Laboratoire des signaux et systèmes (L2S), CNRS, CentraleSupélec, Université Paris-Sud, Université Paris-Saclay, \\ 3, rue Joliot-Curie, 91192 Gif-sur-Yvette, cedex, France \\ ${ }^{\mathrm{c}}$ Univ. Grenoble Alpes/CNRS, GIPSA-Lab, F-38000 Grenoble, France
}

\begin{abstract}
The robust controlled invariance describes the ability to maintain, using suitable control actions, the state of a system in a set for any value of the disturbances. By considering a class of monotone systems and a multidimensional interval as target set, we obtain a simple characterization of the robust controlled invariance. We then give a method to stabilize the state into a robust controlled invariant interval when it is initialized outside of the target set. These results are applied to a model for the temperature control in an intelligent building equipped with automated underfloor air distribution (UFAD) and implemented in a small-scale experimental UFAD flat.
\end{abstract}

Key words: Monotone systems ; robust controlled invariance ; temperature control

\section{Introduction}

For autonomous systems, the notion of positively invariant set describes the property that trajectories initialized in a set remain inside this set forever. An extensive survey on the topic of invariance can be found in [6]. When a control input is used to enforce the invariance, we talk about controlled invariance, independently introduced in [3] and [19]. An overview of the uses and results on controlled invariant sets for linear systems is given in [17]. In this paper, we are interested in the study of robust controlled invariance where the robustness refers to bounded external disturbances.

In this paper, we deal with a class of nonlinear systems satisfying a monotonicity property. Monotone systems are systems which preserve partial orderings on the states, see [15] for autonomous systems and [2] for controlled systems. We show that this monotonicity prop-

\footnotetext{
* This work was supported by a $\mathrm{PhD}$ scholarship and the research project COHYBA funded by Région Rhône-Alpes. Corresponding author: P.-J. Meyer.

Email addresses: pjmeyer@kth.se (Pierre-Jean Meyer), Antoine.Girard@12s . centralesupelec.fr (Antoine Girard), Emmanuel.Witrant@ujf-grenoble.fr (Emmanuel Witrant).
}

erty, associated with simple sets (multidimensional intervals), can be used to obtain a characterization for the robust controlled invariance, using only the extremal values of each state, control and disturbance input. We also show how these robust controlled invariant sets can be used to synthesize robust stabilizing controllers for monotone control systems. To the knowledge of the authors, there are very few works on (controlled) invariance of monotone nonlinear systems: invariance of intervals for autonomous monotone systems has been considered in [1]; methods for approximating the maximal controlled invariant set for monotone discrete time systems without disturbance are presented in [11]; a controller for reference tracking in a monotone SISO system is synthesized under state constraints in [8]; finally, robust controlled invariance are considered for a class of monotone systems with planar outputs in [9]. Monotone systems can be found in numerous fields such as molecular biology [16], chemical networks [5], multi-vehicle systems [10], or thermal dynamics in buildings, which is the application considered in this paper. We consider an underfloor air distribution (UFAD) system based on a 4-room small-scale experiment of a flat. We apply the results developed in the paper to that system and report the results obtained on our experimental platform.

The paper is organized as follows. In Section 2, we intro- 
duce the class of systems we consider. In Section 3, we establish a certain number of results on robust invariance and robust controlled invariance. In Section 4, we show how our characterization of robust controlled invariant interval allows us to synthesize robust stabilizing controllers. Finally these methodological results are applied to the temperature control of a UFAD model and tested on a small-scale experimental flat in Section 5 .

\section{Monotone control systems}

We consider a class of nonlinear systems given by:

$$
\dot{x}=f(x, u, w),
$$

where $x \in \mathbb{R}^{n}, u \in \mathbb{R}^{p}$ and $w \in \mathbb{R}^{q}$ denote the state, the control input and the disturbance input, respectively. The vector field $f$ is assumed to be locally Lipschitz. The trajectories of (1) are denoted by $\Phi\left(\cdot, x_{0}, \mathbf{u}, \mathbf{w}\right)$ where $\Phi\left(t, x_{0}, \mathbf{u}, \mathbf{w}\right)$ is the state reached at time $t \in \mathbb{R}_{0}^{+}$ from the initial state $x_{0} \in \mathbb{R}^{n}$, under control and disturbance inputs $\mathbf{u}: \mathbb{R}_{0}^{+} \rightarrow \mathbb{R}^{p}$ and $\mathbf{w}: \mathbb{R}_{0}^{+} \rightarrow \mathbb{R}^{q}$. When the control inputs of system (1) are generated by a state-feedback controller $\mathrm{u}: \mathbb{R}^{n} \rightarrow \mathbb{R}^{p}$, the dynamics of the closed-loop system is given by $\dot{x}=f_{\mathrm{u}}(x, w)=f(x, \mathrm{u}(x), w)$ and its trajectories are denoted by $\Phi_{\mathrm{u}}\left(\cdot, x_{0}, \mathbf{w}\right)$.

\subsection{Monotonicity}

The subsequent developments of this paper require the system (1) to satisfy some monotonicity property and we particularly focus on the subclass of cooperative systems. For a variable $z \in\{x, u, w\}$ with $z \in \mathbb{R}^{m}$, the partial orderings $\succeq_{z}, \preceq_{z}, \gg_{z}$ and $\ll_{z}$ represent the classical componentwise inequalities $\geq, \leq,>$ and $<$ on $\mathbb{R}^{m}$. These orderings can be extended to functions $\mathbf{z}, \mathbf{z}^{\prime}: \mathbb{R}_{0}^{+} \rightarrow \mathbb{R}^{m}$ where $\mathbf{z} \succeq_{z} \mathbf{z}^{\prime}$ if and only if $\mathbf{z}(t) \succeq_{z} \mathbf{z}^{\prime}(t)$ for all $t \geq 0$. Given $\underline{z}$ and $\bar{z} \in \mathbb{R}^{m}$ with $\bar{z} \succeq_{z} \underline{z},[\underline{z}, \bar{z}]$ denotes the interval such that $z \in[\underline{z}, \bar{z}]$ if and only if $\bar{z} \succeq_{z} z \succeq_{z} \underline{z}$. Following [2], we now introduce the notion of cooperative system using the partial orderings $\succeq_{x}, \succeq_{u}$ and $\succeq_{w}$.

Definition 1 (Cooperative system) System (1) is cooperative if for all $x \succeq_{x} x^{\prime}, \mathbf{u} \succeq_{u} \mathbf{u}^{\prime}, \mathbf{w} \succeq_{w} \mathbf{w}^{\prime}$, it holds for all $t \geq 0, \Phi(t, x, \mathbf{u}, \mathbf{w}) \succeq_{x} \Phi\left(t, x^{\prime}, \mathbf{u}^{\prime}, \mathbf{w}^{\prime}\right)$.

In a cooperative system, a variable (state or input) affects a state always in a positive way, as shown by the following characterization which is a generalization of the Kamke condition to systems with inputs.

Proposition 2 [2] System (1) is cooperative if and only if for all $i \in\{1, \ldots, n\}$, for all $x \succeq_{x} x^{\prime}$ with $x_{i}=x_{i}^{\prime}$, $u \succeq_{u} u^{\prime}, w \succeq_{w} w^{\prime}$, it holds $f_{i}(x, u, w) \geq f_{i}\left(x^{\prime}, u^{\prime}, w^{\prime}\right)$.

In the following, we shall make the following assumption for system (1).
Assumption 3 System (1) is cooperative with bounded control and disturbance inputs: $u \in[\underline{u}, \bar{u}]$ and $w \in[\underline{w}, \bar{w}]$.

Assumption 3 is crucial for our robustness analysis since we can focus on studying the behavior of the system only for the extremal values of the variables: all other behaviors are necessarily bounded by the extremal behaviors.

\subsection{Additional assumptions}

Some of the results presented in the following sections need additional requirements on system (1). The following assumption is necessary for all main results presented in this paper.

Assumption 4 System (1) satisfies the local control property: any component of the control input directly influences a single component of the state in (1).

With this assumption, system (1) can then be written as $\dot{x}_{i}=f_{i}\left(x, u_{i}, w\right)$ for all $i \in\{1, \ldots, n\}$, where $u_{i}$ represents all input components with a direct influence on $x_{i}$ (i.e. $u_{i}$ can be a vector, a scalar or the empty set).

We also extend the definition of a static input-state characteristic introduced in [2] to systems with both control and disturbance inputs. The following assumption is optional as it is only useful for secondary results: the main results can still be applied if it is not satisfied.

Assumption 5 System (1) has a static input-state characteristic $k_{x}: \mathbb{R}^{p} \times \mathbb{R}^{q} \rightarrow \mathbb{R}^{n}$ : for each pair $(u, w)$ of constant control and disturbance inputs, (1) has a unique globally asymptotically stable equilibrium $k_{x}(u, w)$.

\section{Robust invariance for monotone systems}

In this section, we present and characterize several notions of robust invariance and focus on finding the associated inputs and state intervals. Some of the results of this section were previously presented in [13] with less generality.

\subsection{Robust invariance}

A robust invariant is a set such that if the state of the system is initialized in this set then it remains in the set forever, for all values of the control and disturbance inputs. Restricting this notion to intervals, we have the following definition.

Definition 6 (Robust invariance) An interval $[\underline{x}, \bar{x}]$ is robust invariant if, for all $x_{0} \in[\underline{x}, \bar{x}], \mathbf{u} \in[\underline{u}, \bar{u}], \mathbf{w} \in$ $[\underline{w}, \bar{w}]$, it holds for all $t \geq 0, \Phi\left(t, x_{0}, \mathbf{u}, \mathbf{w}\right) \in[\underline{x}, \bar{x}]$. 
Thus, if the initial state is in a robust invariant interval, this interval contains all reachable states from this initial condition. However, this does not mean that all points in this interval are reachable. In order to minimize the quantity of non-reachable states in the interval, one can look for the minimal robust invariant interval (where minimality refers to the set inclusion), which is useful in the subsequent study as we can restrict the analysis of system (1) to that region.

Theorem 7 Under Assumption 3, $[\underline{x}, \bar{x}]$ is robust invariant if and only if $f(\bar{x}, \bar{u}, \bar{w}) \preceq_{x} 0$ and $f(\underline{x}, \underline{u}, \underline{w}) \succeq_{x} 0$. In addition, if Assumption 5 holds, then the minimal robust invariant interval is $\left[k_{x}(\underline{u}, \underline{w}), k_{x}(\bar{u}, \bar{w})\right]$.

PROOF. $[\underline{x}, \bar{x}]$ is robust invariant if and only if for any element $x$ of the boundary of $[\underline{x}, \bar{x}]$, the flow $\Phi(t, x, \mathbf{u}, \mathbf{w})$ does not leave the interval. This is equivalent to having the vector field at $x$ point inside the interval for all $u \in[\underline{u}, \bar{u}]$ and $w \in[\underline{w}, \bar{w}]$. By considering the elements of the boundary $\underline{x}$ and $\bar{x}$, it is clear that the conditions above are necessary. Let us show that they are also sufficient under Assumption 3. By Proposition 2, we have for all $i \in\{1, \ldots, n\}, u \in[\underline{u}, \bar{u}], w \in[\underline{w}, \bar{w}]$ and $x \in[\underline{x}, \bar{x}]$ with $x_{i}=\bar{x}_{i}, f_{i}(x, u, w) \leq f_{i}(\bar{x}, \bar{u}, \bar{w}) \leq 0$ and for all $i \in\{1, \ldots, n\}, u \in[\underline{u}, \bar{u}], w \in[\underline{w}, \bar{w}]$ and $x \in[\underline{x}, \bar{x}]$ with $x_{i}=\underline{x}_{i}, f_{i}(x, u, w) \geq f_{i}(\underline{x}, \underline{u}, \underline{w}) \geq 0$. Therefore, $[\underline{x}, \bar{x}]$ is robust invariant. Now, let us assume that Assumption 5 holds. By definition, we have $f\left(k_{x}(\bar{u}, \bar{w}), \bar{u}, \bar{w}\right)=0$ and $f\left(k_{x}(\underline{u}, \underline{w}), \underline{u}, \underline{w}\right)=0$. From what precedes, $\left[k_{x}(\underline{u}, \underline{w}), k_{x}(\bar{u}, \bar{w})\right]$ is robust invariant. Also, any robust invariant interval would contain $k_{x}(\underline{u}, \underline{w})$ and $k_{x}(\bar{u}, \bar{w})$ as these are globally asymptotically stable equilibria for constant inputs $\mathbf{u}=\underline{u}, \mathbf{w}=\underline{w}$ and $\mathbf{u}=\bar{u}$ and $\mathbf{w}=\bar{w}$, respectively. Hence, the robust invariant interval $\left[k_{x}(\underline{u}, \underline{w}), k_{x}(\bar{u}, \bar{w})\right]$ is minimal with respect to set inclusion.

Note that in the absence of Assumption 5, there may not exist a minimal robust invariant interval.

\subsection{Robust controlled invariance}

For the definition of robust controlled invariance, we now take advantage of the control input $u$ to counteract the influence of the disturbance $w$.

\section{Definition 8 (Robust controlled invariance)}

Interval $[\underline{x}, \bar{x}]$ is robust controlled invariant if there exists a controller $\mathrm{u}:[\underline{x}, \bar{x}] \rightarrow[\underline{u}, \bar{u}]$ such that for all $x_{0} \in[\underline{x}, \bar{x}], \quad \mathbf{w} \in[\underline{w}, \bar{w}]$, it holds for all $t \geq 0$, $\Phi_{\mathrm{u}}\left(t, x_{0}, \mathbf{w}\right) \in[\underline{x}, \bar{x}]$. We call $\mathrm{u}$ an invariance controller in $[\underline{x}, \bar{x}]$.

The following result characterizes robust controlled invariant intervals based on the sign of the vector field $f$.
Theorem 9 Under Assumptions 3 and $4,[\underline{x}, \bar{x}]$ is robust controlled invariant if and only if $f(\bar{x}, \underline{u}, \bar{w}) \preceq_{x} 0$ and $f(\underline{x}, \bar{u}, \underline{w}) \succeq_{x} 0$.

PROOF. We prove necessity by contrapositive. Assume that there exists $i \in\{1, \ldots, n\}$ such that $f_{i}(\bar{x}, \underline{u}, \bar{w})>0$. By Proposition 2, it follows that $\forall u \in[\underline{u}, \bar{u}], f_{i}(\bar{x}, u, \bar{w}) \geq f_{i}(\bar{x}, \underline{u}, \bar{w})>0$. Thus no value of the control input $u$ can make the vector field at $\bar{x}$ point inside the interval, making it non-invariant. We can have a similar reasoning if there exists $i \in\{1, \ldots, n\}$ such that $f_{i}(\underline{x}, \bar{u}, \underline{w})<0$. Let us now prove sufficiency. By Assumption 4, we have that for all $i \in\{1, \ldots, n\}$, $f_{i}(x, u, w)=f_{i}\left(x, u_{i}, w\right)$ with independent inputs $u_{i} \in\left[\underline{u}_{i}, \bar{u}_{i}\right]$. Then, by Proposition 2 , we have that for all $i \in\{1, \ldots, n\}, w \in[\underline{w}, \bar{w}]$ and $x \in[\underline{x}, \bar{x}]$ with $x_{i}=\bar{x}_{i}, f_{i}\left(x, \underline{u}_{i}, w\right) \leq f_{i}\left(\overline{\bar{x}}, \underline{u}_{i}, \bar{w}\right) \leq 0$ and for all $i \in\{1, \ldots, n\}, w \in[\underline{w}, \bar{w}]$ and $x \in[\underline{x}, \bar{x}]$ with $x_{i}=\underline{x}_{i}$, $f_{i}\left(x, \bar{u}_{i}, w\right) \geq f_{i}\left(\underline{x}, \bar{u}_{i}, \underline{w}\right) \geq 0$. Since the $u_{i}$ are independent, it follows from the previous inequalities, that for any state $x$ on the boundary of the interval $[\underline{x}, \bar{x}]$, there exists a value of the control input $\mathrm{u}(x) \in[\underline{u}, \bar{u}]$ such that the vector field at $x$ points inside the interval for any value of the disturbance. Using such controller $u$, we can always force the flow toward the interior when the state reaches the boundary of the interval. This implies the robust controlled invariance of the interval.

Theorem 9 states that if the extremal values of the controller can maintain the system in $[\underline{x}, \bar{x}]$ for the extremal values of the disturbances, then the invariance in the interval is satisfied. Let us remark that in the absence of Assumption 4, the conditions are still necessary but not sufficient. We now give a characterization of the invariance controllers, where $u_{i}$ denotes the vector of all input components with a direct influence on the state $x_{i}$.

Proposition 10 Under Assumptions 3 and 4 , let $[\underline{x}, \bar{x}]$ be a robust controlled invariant. A controller $\mathrm{u}:[\underline{x}, \bar{x}] \rightarrow$ $[\underline{u}, \bar{u}]$ is an invariance controller in $[\underline{x}, \bar{x}]$ if and only if for all $i \in\{1, \ldots, n\}$ :

$$
\begin{gathered}
\mathrm{u}_{i}(x) \in \begin{cases}\underline{U_{i}}(x) & \text { if } x_{i}=\overline{x_{i}}, \\
{\left[\underline{u_{i}}, \overline{u_{i}}\right]} & \text { if } x_{i} \in\left(\underline{x_{i}}, \overline{x_{i}}\right), \\
\overline{U_{i}}(x) \quad \text { if } x_{i}=\underline{x_{i}}\end{cases} \\
\text { where } \underline{U}_{i}(x)=\left\{u_{i} \in\left[\underline{u_{i}}, \overline{u_{i}}\right] \mid f_{i}\left(x, u_{i}, \bar{w}\right) \leq 0\right\}, \\
\bar{U}_{i}(x)=\left\{u_{i} \in\left[\underline{u_{i}}, \overline{u_{i}}\right] \mid f_{i}\left(x, u_{i}, \underline{w}\right) \geq 0\right\} .
\end{gathered}
$$

PROOF. It is necessary and sufficient that for all $x$ on the boundary of the interval $[\underline{x}, \bar{x}]$, the vector field $f_{\mathrm{u}}$ of the closed-loop $\dot{x}=f_{\mathrm{u}}(x, w)$ at $x$ points inside the interval for all values of the disturbance. From Assumption 4, this is the case if and only if $f_{i}\left(x, \mathbf{u}_{i}(x), w\right) \leq 0$ (respectively $\left.f_{i}\left(x, \mathbf{u}_{i}(x), w\right) \geq 0\right)$ for all $w \in[\underline{w}, \bar{w}]$ whenever a state component $x_{i}$ reaches $\bar{x}_{i}$ (respectively $\underline{x}_{i}$ ). Using 
the monotonicity of $f_{i}$ with respect to the disturbance $w$, we obtain the conditions given in the proposition.

Since $[\underline{x}, \bar{x}]$ is a robust controlled invariant, it is easy to show from Theorem 9 and Proposition 2 that for all $x \in[\underline{x}, \bar{x}]$ such that $x_{i}=\overline{x_{i}}$ (respectively $x_{i}=x_{i}$ ), we have $\underline{u}_{i} \in \underline{U}_{i}(x)$ (respectively $\overline{u_{i}} \in \bar{U}_{i}(x)$ ). Then, the necessary and sufficient conditions given by (2) admit a very simple realization:

$$
\mathrm{u}_{i}(x)=\underline{u_{i}}+\left(\overline{u_{i}}-\underline{u_{i}}\right) \frac{\overline{x_{i}}-x_{i}}{\overline{x_{i}}-\underline{x_{i}}} .
$$

Let us remark that this controller is affine and decentralized in the sense that the value of input $\mathrm{u}_{i}(x)$ only depends on state component $x_{i}$. Then $\mathrm{u}_{i}(x)=\mathrm{u}_{i}\left(x^{\prime}\right)$ when $x_{i}=x_{i}^{\prime}$ and Proposition 2 implies that the closedloop system $\dot{x}=f_{\mathrm{u}}(x, w)$ with controller (3) is cooperative. Indeed, for all $x \succeq_{x} x^{\prime}, x_{i}=x_{i}^{\prime}, w \succeq_{w} w^{\prime}$, it holds $f_{\mathrm{u} i}(x, w) \geq f_{\mathrm{u} i}\left(x^{\prime}, w^{\prime}\right)$. Note that in Proposition 10, no assumption on the regularity of the controllers is needed and we can also consider discontinuous realizations of (2) such as a classical bang-bang controller.

\subsection{Robust local stabilizability}

In this section we introduce the notion of robust local stabilizability, which is closely related to the results on robust controlled invariance presented in Section 3.2.

Definition 11 (Robust local stabilizability) The state $x^{*}$ is robustly locally stabilizable if for all $\varepsilon>0$, there exist $\delta>0$ and $\mathrm{u}: \mathcal{B}\left(x^{*}, \varepsilon\right) \rightarrow[\underline{u}, \bar{u}]$ such that for all $x_{0} \in \mathcal{B}\left(x^{*}, \delta\right), \mathbf{w} \in[\underline{w}, \bar{w}]$, it holds for all $t \geq 0$ $\Phi_{\mathbf{u}}\left(t, x_{0}, \mathbf{w}\right) \in \mathcal{B}\left(x^{*}, \varepsilon\right)$, where $\mathcal{B}\left(x^{*}, r\right)$ denotes the ball of radius $r$ centered at $x^{*}$.

Definition 11 can be explained as follows: the target state $x^{*}$ is robustly locally stabilizable if for any small ball around the state $x^{*}$, there exists another ball of initial states such that the system can be robustly controlled to stay in the first ball. Thus with a minor modification, the robust local stabilizability of $x^{*}$ can be obtained with small robust controlled invariant intervals around $x^{*}$. This consideration leads to the following result.

Theorem 12 Under Assumptions 3 and $4, x^{*}$ is robustly locally stabilizable if $f\left(x^{*}, \underline{u}, \bar{w}\right) \ll_{x} \quad 0$ and $f\left(x^{*}, \bar{u}, \underline{w}\right) \gg_{x} 0$. If $x^{*}$ is robustly locally stabilizable, these conditions are satisfied with non-strict inequalities.

PROOF. For the first implication, we choose a ball $\mathcal{B}\left(x^{*}, \varepsilon\right)$ of radius $\varepsilon$ centered on $x^{*}$. Using the continuity of $f$, there exist two states $x, \bar{x} \in \mathcal{B}\left(x^{*}, \varepsilon\right)$ with $\underline{x} \ll_{x} x^{*}$ and $\bar{x} \gg_{x} x^{*}$ such that $f(\bar{x}, \underline{u}, \bar{w}) \ll_{x} 0$ and $f(\underline{x}, \bar{u}, \underline{w}) \gg_{x} 0$. Thus $[\underline{x}, \bar{x}] \subseteq \mathcal{B}\left(x^{*}, \varepsilon\right)$ is a robust controlled invariant interval as in Definition 8 and we then obtain Definition 11 by choosing $\delta$ such that the ball of initial states $\mathcal{B}\left(x^{*}, \delta\right) \subseteq[\underline{x}, \bar{x}]$. We prove the second part of the theorem by contrapositive. We assume that there exists $i \in\{1, \ldots, n\}$ such that $f_{i}\left(x^{*}, \underline{u}, \bar{w}\right)>0$. Using the continuity of $f$, we can choose $\varepsilon>0$ such that for all $x \in \mathcal{B}\left(x^{*}, \varepsilon\right), f_{i}(x, \underline{u}, \bar{w})>0$. If we take $w=\bar{w}$, then we can use Proposition 2 to extend this inequality to any $u$ : for all $u \in[\underline{u}, \bar{u}], x \in \mathcal{B}\left(x^{*}, \varepsilon\right), f_{i}(x, u, \bar{w})>0$. This means that if the state is in $\mathcal{B}\left(x^{*}, \varepsilon\right)$ and $w=\bar{w}$, then for any value of the control input the trajectory of the system will leave $\mathcal{B}\left(x^{*}, \varepsilon\right)$. This implies that $x^{*}$ is not robustly locally stabilizable. This results is similarly obtained if we initially assume that there exists $i \in\{1, \ldots, n\}$ such that $f_{i}\left(x^{*}, \bar{u}, \underline{w}\right)<0$.

\section{Robust stabilization}

In the previous section, we have addressed the problem of synthesizing a controller in order to maintain the state of system (1) in a given interval. The next step is to synthesize a controller that will bring the state in this interval if the initial state lies outside the interval.

Let $\left[x_{0}, \overline{x_{0}}\right]$ be an interval of initial states and let $\left[x_{f}, \overline{x_{f}}\right] \subseteq\left[x_{0}, \overline{x_{0}}\right]$ (with $x_{0} \ll_{x} x_{f}$ and $\overline{x_{f}} \ll_{x} \overline{x_{0}}$ ) be the target interval, i.e. the interval where we should steer the state of (1).

Definition 13 A controller $\mathrm{u}:\left[x_{0}, \overline{x_{0}}\right] \rightarrow[\underline{u}, \bar{u}]$ is said to be a stabilizing controller from $\left[\underline{x_{0}}, \overline{x_{0}}\right]$ to $\left[x_{f}, \overline{x_{f}}\right]$, if for all $x_{0} \in\left[x_{0}, \overline{x_{0}}\right], \mathbf{w} \in[\underline{w}, \bar{w}]$, there exists $T \overline{\geq} 0$, such that for all $t \geq T$, it holds $\bar{\Phi}_{\mathbf{u}}\left(t, x_{0}, \mathbf{w}\right) \in\left[\underline{x_{f}}, \overline{x_{f}}\right]$.

Note that in Definition 13 we are interested in the set stability in finite time and not in its asymptotic stability. We aim to synthesize stabilizing controllers under the following assumption.

Assumption 14 There exist continuously differentiable functions $\underline{X}:[0,1] \rightarrow \mathbb{R}^{n}$ and $\bar{X}:[0,1] \rightarrow \mathbb{R}^{n}$, respectively strictly decreasing and increasing with $\frac{d X}{d \lambda}(\lambda) \ll_{x} 0$ and $\frac{d \bar{X}}{d \lambda}(\lambda) \gg_{x} 0$ for all $\lambda \in[0,1]$, such that $\underline{X}(0)=x_{f}$, $\underline{X}(1)=\underline{x_{0}}, \bar{X}(0)=\overline{x_{f}}, \bar{X}(1)=\overline{x_{0}}$ and for all $\lambda \in[0, \overline{1]}$, $f(\underline{X}(\lambda), \bar{u}, \underline{w}) \gg_{x} 0, f(\bar{X}(\lambda), \underline{u}, \bar{w}) \ll_{x} 0$.

\subsection{Choice of the functions $\underline{X}$ and $\bar{X}$}

Assumption 14 and Theorem 9 imply that for all $\lambda, \lambda^{\prime} \in$ $[0,1]$, the interval $\left[\underline{X}(\lambda), \bar{X}\left(\lambda^{\prime}\right)\right]$ is a robust controlled invariant. The main idea of our approach is to use this parameterized family of robust controlled invariants to drive the state to $\left[x_{f}, \overline{x_{f}}\right]$. Two examples of candidates for such functions $\underline{X}$ and $\bar{X}$ are presented below. 
The first choice consists in simple linear functions: $\underline{X}(\lambda)=\lambda \underline{x_{0}}+(1-\lambda) x_{f}$ and $\bar{X}(\lambda)=\lambda \overline{x_{0}}+(1-\lambda) \overline{x_{f}}$. The first part of Assumption 14 clearly holds. It remains to check that the second part holds for all $\lambda \in[0,1]$. This is always the case if the sets $\left\{x \in \mathbb{R}^{n} \mid f(x, \bar{u}, \underline{w}) \gg_{x} 0\right\}$ and $\left\{x \in \mathbb{R}^{n} \mid f(x, \underline{u}, \bar{w}) \ll_{x} 0\right\}$ are convex. Otherwise, the condition can easily be verified numerically.

For the second possible choice, let us assume that Assumption 5 holds and that there exist $\underline{u_{0}}, u_{f}, \overline{u_{0}}, \overline{u_{f}} \in$ $[\underline{u}, \bar{u}]$ (with $\underline{u_{0}} \ll_{u} u_{f} \ll_{u} \bar{u}$ and $\underline{u} \ll_{u} \overline{\overline{u_{f}}} \overline{\ll_{u}} \overline{u_{0}}$ ) such that $\underline{x_{0}}=\overline{k_{x}}\left(\underline{u_{0}}, \underline{w}\right), x_{f}=k_{x}\left(u_{f}, \underline{w}\right), \overline{x_{0}}=k_{x}\left(\overline{u_{0}}, \bar{w}\right)$, and $\overline{\overline{x_{f}}}=k_{x}\left(\overline{u_{f}}, \bar{w}\right)$. Let

$$
\begin{gathered}
\underline{U}(\lambda)=\lambda \underline{u_{0}}+(1-\lambda) \underline{u_{f}}, \underline{X}(\lambda)=k_{x}(\underline{U}(\lambda), \underline{w}) \\
\bar{U}(\lambda)=\lambda \overline{u_{0}}+(1-\lambda) \overline{u_{f}}, \bar{X}(\lambda)=k_{x}(\bar{U}(\lambda), \bar{w}) .
\end{gathered}
$$

We can then show the following result.

Proposition 15 Under Assumptions 3, 4 and 5, let us assume that:

- $f$ is continuously differentiable, for all $x \in \mathbb{R}^{n}, u \in$ $\mathbb{R}^{p}, w \in \mathbb{R}^{q}$

- the matrix of partial derivatives $\partial f / \partial x$ is invertible;

- $\partial f_{i} / \partial u_{i}>0$, for all $i \in\{1, \ldots, n\}$.

Then, the functions defined by (4) satisfy Assumption 14.

PROOF. It is shown in [2] that $k_{x}$ is monotone. It is thus straightforward to show that $\underline{X}$ and $\bar{X}$ are decreasing and increasing, respectively. Moreover, note that $f(\underline{X}(\lambda), \underline{U}(\lambda), \underline{w})=0$. By the implicit function theorem it follows that $\underline{X}$ is continuously differentiable and that

$$
\frac{\partial f}{\partial x} \times \frac{d \underline{X}}{d \lambda}(\lambda)=-\frac{\partial f}{\partial u} \times \frac{d \underline{U}}{d \lambda}(\lambda)
$$

where the partial derivatives are evaluated at $\underline{X}(\lambda)$, $\underline{U}(\lambda), \underline{w}$. Assumptions 3 and 4 imply that for all $i \in\{1, \ldots, n\}$

$$
\sum_{j=1}^{n} \frac{\partial f_{i}}{\partial x_{j}} \frac{d \underline{X}_{j}}{d \lambda}(\lambda)=-\frac{\partial f_{i}}{\partial u_{i}} \frac{d \underline{U}_{i}}{d \lambda}(\lambda)>0 .
$$

Then, $\underline{X}$ decreasing and Assumption 3 yields

$$
\frac{\partial f_{i}}{\partial x_{i}} \frac{d \underline{X}_{i}}{d \lambda}(\lambda)>-\sum_{j \neq i} \frac{\partial f_{i}}{\partial x_{j}} \frac{d \underline{X}_{j}}{d \lambda}(\lambda) \geq 0
$$

which implies that $\frac{d \underline{X}_{i}}{d \lambda}(\lambda) \neq 0$, hence $\underline{X}$ is strictly decreasing. Similarly, we can show that $\bar{X}$ is continuously differentiable and strictly increasing: the first part of Assumption 14 thus holds. For the second part, $\partial f_{i} / \partial u_{i}>$ 0 and $\underline{U}(\lambda) \ll_{u} \bar{u}$ give for all $\lambda \in[0,1]$ :

$$
f(\underline{X}(\lambda), \bar{u}, \underline{w}) \gg_{x} f(\underline{X}(\lambda), \underline{U}(\lambda), \underline{w})=0 .
$$
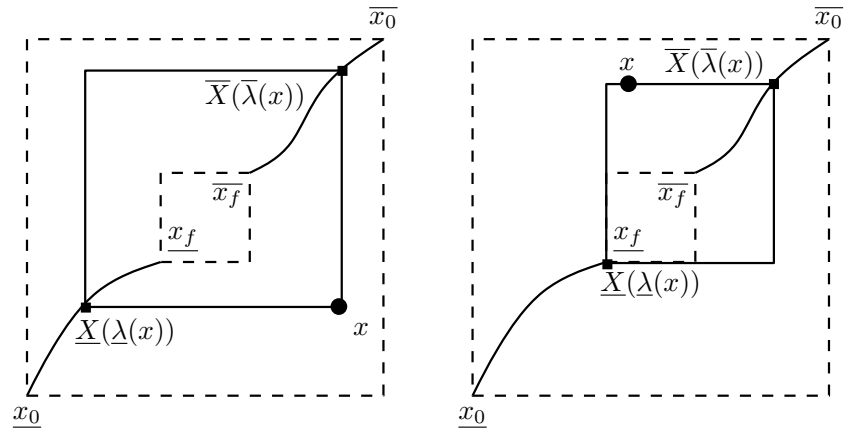

Fig. 1. Smallest elements of the parameterized family of robust controlled invariants $\left[\underline{X}(\lambda), \bar{X}\left(\lambda^{\prime}\right)\right]$ containing state $x$.

A similar fact holds for $\bar{X}$.

\subsection{Stabilizing controller synthesis}

Let us assume that Assumption 14 holds, and define the functions $\underline{\lambda}, \bar{\lambda}:\left[\underline{x_{0}}, \overline{x_{0}}\right] \rightarrow[0,1]$ given by

$$
\begin{aligned}
& \underline{\lambda}(x)=\min \left\{\lambda \in[0,1] \mid \underline{X}(\lambda) \preceq_{x} x\right\}, \\
& \bar{\lambda}(x)=\min \left\{\lambda \in[0,1] \mid \bar{X}(\lambda) \succeq_{x} x\right\} .
\end{aligned}
$$

In other words, $[\underline{X}(\underline{\lambda}(x)), \bar{X}(\bar{\lambda}(x))]$ is the smallest interval of the parameterized family $\left[\underline{X}(\lambda), \bar{X}\left(\lambda^{\prime}\right)\right]$ containing $x$, as illustrated in Figure 1 . The main idea of our stabilization approach is to use a controller $\mathrm{u}$ that renders each interval $[\underline{X}(\underline{\lambda}(x)), \bar{X}(\bar{\lambda}(x))]$ invariant and makes $\underline{\lambda}(x)$ and $\bar{\lambda}(x)$ act like Lyapunov functions to show that the state reaches the target interval $\left[x_{f}, \overline{x_{f}}\right]=$ $[\underline{X}(0), \bar{X}(0)]$ in finite time.

Theorem 16 Under Assumptions 3, 4 and 14, the controller u defined by (5) and

$$
\mathrm{u}_{i}(x)=\underline{u_{i}}+\left(\overline{u_{i}}-\underline{u_{i}}\right) \frac{\bar{X}_{i}(\bar{\lambda}(x))-x_{i}}{\bar{X}_{i}(\bar{\lambda}(x))-\underline{X}_{i}(\underline{\lambda}(x))} .
$$

is a stabilizing controller from $\left[\underline{x_{0}}, \overline{x_{0}}\right]$ to $\left[\underline{x_{f}}, \overline{x_{f}}\right]$.

PROOF. From Assumption 14, there exists $\alpha>0$ such that for all $i \in\{1, \ldots, n\}, \lambda \in[0,1]$,

$$
f_{i}(\bar{X}(\lambda), \underline{u}, \bar{w}) \leq-\alpha
$$

Since $\bar{X}$ is strictly increasing with $\frac{d \bar{X}}{d \lambda}(\lambda) \gg_{x} 0$ and continuously differentiable, then $\bar{X}_{i}^{-1}$ is well defined, strictly increasing and continuously differentiable on $\left[\bar{x}_{f}, \bar{x}_{0} i\right]$. Then there exists $\beta>0$ such that for all $i \in\{1, \ldots, n\}$,

$$
\forall x_{i} \in\left[\bar{x}_{f}, \bar{x}_{0}\right], \frac{d}{d x_{i}} \bar{X}_{i}^{-1}\left(x_{i}\right) \geq \beta
$$


Although it is not necessary for the robust stabilization, to simplify the notations of the proof we assume that the domain of definition of the function $\bar{X}_{i}^{-1}$ can be extended to $\left[\underline{x}_{0}, \bar{x}_{0} i\right]$ while keeping its properties of continuous differentiability and monotonicity. This means that $\bar{X}_{i}^{-1}$ takes negative values for $x_{i}<\bar{x}_{f}$. Then, if we introduce $\bar{\lambda}_{i}: \mathbb{R}^{n} \rightarrow[0,1]$ such that $\bar{\lambda}_{i}(x)=\bar{X}_{i}^{-1}\left(x_{i}\right)$ and $\bar{\lambda}_{0}$ a constant function equal to zero, the function $\bar{\lambda}$ can now be written as the maximum of continuously differentiable functions: $\bar{\lambda}(x)=\max _{i \in\{0, \ldots, n\}}\left(\bar{\lambda}_{i}(x)\right)$.

Let $\overline{\mathcal{I}}(x)=\left\{i \in\{0, \ldots, n\} \mid \bar{\lambda}_{i}(x)=\bar{\lambda}(x)\right\}$. Let $x_{0} \in$ $\left[\underline{x_{0}}, \overline{x_{0}}\right], \mathbf{w} \in[\underline{w}, \bar{w}], \mathbf{x}=\Phi_{\mathbf{u}}\left(., x_{0}, \mathbf{w}\right), t \in \mathbb{R}_{0}^{+}$and $i \in$ $\overline{\mathcal{I}}(\mathbf{x}(t)) \backslash\{0\}$. We have

$$
\frac{d \bar{\lambda}_{i}}{d t}(\mathbf{x}(t))=\frac{d \bar{X}_{i}^{-1}}{d x_{i}}\left(\mathbf{x}_{i}(t)\right) * f_{i}\left(\mathbf{x}(t), \mathbf{u}_{i}(\mathbf{x}(t)), \mathbf{w}(t)\right)
$$

Since $i \in \overline{\mathcal{I}}(\mathbf{x}(t))$, we have $\mathbf{x}_{i}(t)=\bar{X}_{i}(\bar{\lambda}(\mathbf{x}(t)))$ and we can use (6), Proposition 2 and (7) to obtain:

$$
f_{i}\left(\mathbf{x}(t), \mathbf{u}_{i}(\mathbf{x}(t)), \mathbf{w}(t)\right) \leq f_{i}\left(\bar{X}(\bar{\lambda}(\mathbf{x}(t))), \underline{u}_{i}, \bar{w}\right) \leq-\alpha .
$$

Inequalities (8) then imply that $\frac{d}{d t} \bar{\lambda}_{i}(\mathbf{x}(t)) \leq-\alpha \beta$, for all $i$ in $\overline{\mathcal{I}}(\mathbf{x}(t)) \backslash\{0\}$. Since $\bar{\lambda}(x)=\max _{i \in\{0, \ldots, n\}}\left(\bar{\lambda}_{i}(x)\right)$, where the functions $\bar{\lambda}_{i}$ are continuously differentiable, its upper right Dini derivative is given by [7]:

$$
D^{+} \bar{\lambda}(\mathbf{x}(t))=\max _{i \in \overline{\mathcal{I}}(\mathbf{x}(t))} \frac{d \bar{\lambda}_{i}}{d t}(\mathbf{x}(t)) .
$$

When $\bar{\lambda}(\mathbf{x}(t))>0$, the index 0 is not in $\overline{\mathcal{I}}(\mathbf{x}(t))$ and $\bar{\lambda}$ is strictly decreasing: $D^{+} \bar{\lambda}(\mathbf{x}(t)) \leq-\alpha \beta$. When $\bar{\lambda}(\mathbf{x}(t))=$ 0 , we have $0 \in \overline{\mathcal{I}}(\mathbf{x}(t))$ and $D^{+} \bar{\lambda}(\mathbf{x}(t))=0$, hence if the state is in the target interval, it remains in it. From [7], we have for all $t \in \mathbb{R}_{0}^{+}$:

$$
\bar{\lambda}(\mathbf{x}(t))-\bar{\lambda}(\mathbf{x}(0))=\int_{0}^{t} D^{+} \bar{\lambda}(\mathbf{x}(s)) d s .
$$

If $\bar{\lambda}(\mathbf{x}(s))>0$ for all $s \in[0, t]$, it follows that $\bar{\lambda}(\mathbf{x}(t))-$ $\bar{\lambda}(\mathbf{x}(0)) \leq-\alpha \beta t$ and then $t<\bar{\lambda}(\mathbf{x}(0)) / \alpha \beta$. Hence, there exists $\bar{T} \in[0, \bar{\lambda}(\mathbf{x}(0)) / \alpha \beta]$ such that $\bar{\lambda}(\mathbf{x}(\bar{T}))=0$. Similarly, we can show that there exists $\underline{T} \in[0, \underline{\lambda}(\mathbf{x}(0)) / \alpha \beta]$ such that $\lambda(\mathbf{x}(\underline{T}))=0$. Thus $\mathrm{u}$ is a stabilizing controller with the finite stabilization time $T=\max (\underline{T}, \bar{T})$.

We have presented a particular stabilizing controller given by (6). Even though (6) is based on the affine and decentralized controller (3), this stabilizing controller is neither affine nor decentralized. There exist many other stabilizing controllers: it is for instance sufficient to choose the control input $\mathrm{u}(x)$ such that the functions $\underline{\lambda}(x)$ and $\bar{\lambda}(x)$ defined by (5) are strictly decreasing.

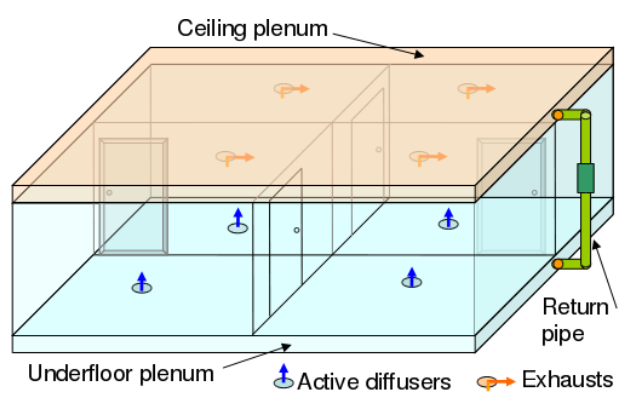

Fig. 2. 4-room flat equipped with underfloor air distribution.

Note that the maximal stabilization time $1 / \alpha \beta$ may be tuned by a suitable choice of $\underline{X}$ and $\bar{X}$ (see (7) and (8)).

\section{Regulation of underfloor air distribution}

The UnderFloor Air Distribution (UFAD) is an alternative solution to traditional ceiling ventilation in buildings, offering improvements in terms of both flexibility and energy consumption [4]. As sketched on Figure 2, a flat equipped with UFAD has an underfloor plenum where the air is cooled down and then sent into each room. The excess of air in the rooms is pushed into the ceiling plenum and sent back to the underfloor plenum to be cooled down again. In this section, we illustrate our previous results on an application of temperature regulation in a flat equipped with UFAD.

\subsection{System description}

The system considered is based on a 4-room small-scale experiment of a flat corresponding to Figure 2. Our focus is the temperature regulation in each room. This is done by acting on the speed of the underfloor fans sending cold air into each room. The system is subject to two types of disturbances. The discrete disturbances are binary variables describing the state of heat sources in each room and of doors between the rooms. The continuous disturbances are all other exogenous inputs: the temperatures of the outside, ceiling plenum and underfloor plenum.

Assuming the uniformity of the temperature in each room, we introduce a 0-dimensional model of the temperature variations, meaning that the variations along the spatial dimensions are neglected. This model is derived from the energy and mass conservation equations in each room $i$ :

$$
\begin{aligned}
\frac{d T_{i}}{d t}= & \sum_{j \in \mathcal{N}_{i}^{*}} a_{i, j}\left(T_{j}-T_{i}\right)+\delta_{s_{i}} b_{i}\left(T_{s_{i}}^{4}-T_{i}^{4}\right) \\
& -c_{i} u_{i}\left(T_{u}-T_{i}\right)+\sum_{j \in \mathcal{N}_{i}} \delta_{d_{i j}} d_{i, j} \max \left(0, T_{j}-T_{i}\right)^{3 / 2}
\end{aligned}
$$

where all constant parameters $a, b, c, d$ are positive, $\mathcal{N}_{i}$ is the set of indices of rooms adjacent to room $i$ and 
Room 1

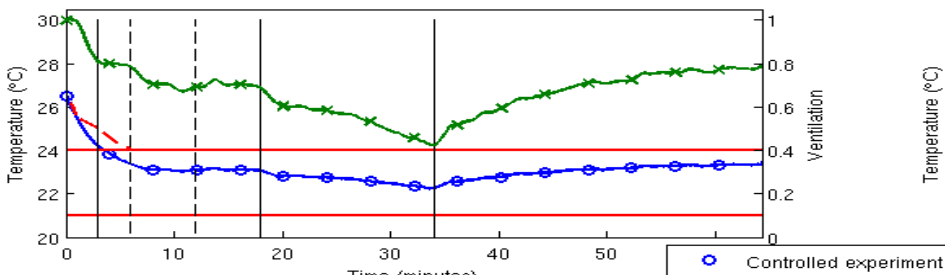

Room 3

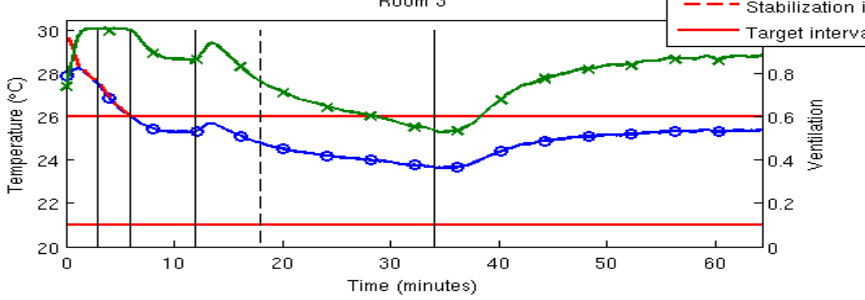

Room 2

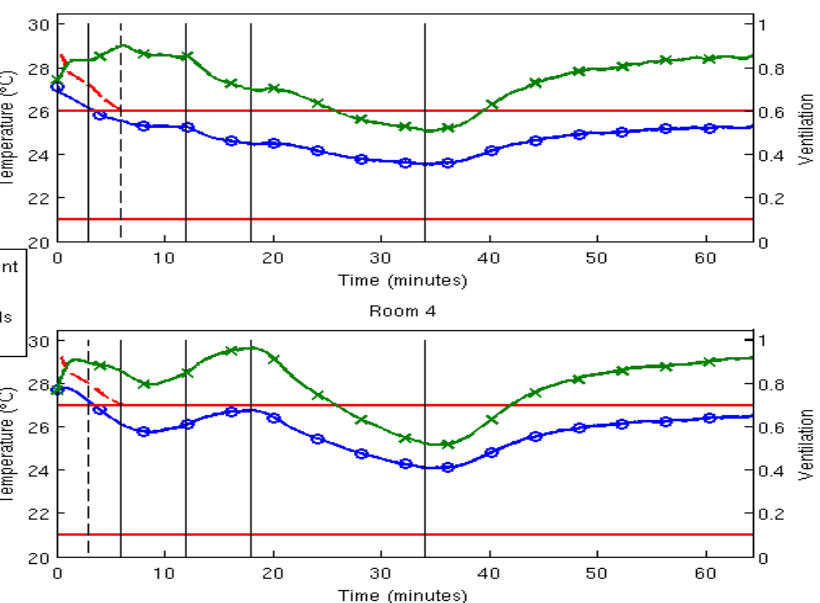

Fig. 3. Room temperature (blue circles), controlled ventilation $(-u \in[0,1]$, green crosses), stabilization intervals (dashed red), target interval (horizontal plain lines) and switching times (vertical lines: plain when related to the room).

$\mathcal{N}_{i}^{*}=\left\{\mathcal{N}_{i}, u, c, o\right\}$ represents all spaces in contact with room $i$ through a wall (neighbor rooms, underfloor, ceiling and outside). The four heat transfers described by (9) are: the thermal conduction through the walls of room $i$, the radiation from a heat source (a lamp in our application) of temperature $T_{s_{i}}$ and binary state $\delta_{s_{i}}$, the controlled ventilation $u_{i} \in[-1,0]$ sending cold air from the underfloor $\left(T_{u}<T_{i}\right)$ and the air flow going through a door when it is open $\left(\delta_{d_{i j}}=1\right)$. A detailed description of the hypotheses used to obtain (9) is given in $[13,18]$.

For the 4-room flat in Figure 2, the dynamics of the whole system are written similarly to $(1): \dot{T}=f(T, u, w, \delta)$, where the four-dimensional vector field $f$ follows $(9)$ on each of its components and depends on the state $T \in \mathbb{R}^{4}$, the control input $u \in \mathbb{R}^{4}$, the exogenous input $w=$ $\left(T_{u}, T_{c}, T_{o}\right)$ considered as a disturbance to our system and the discrete disturbance $\delta \in \mathbb{R}^{8}$ containing one heat source per room and four doors. It is shown in $[13,14,12]$ that this model satisfies Assumptions 3, 4 and 5.

\subsection{Control application}

In this section, we illustrate the robust stabilization (Section 4) and the robust controlled invariance (Section 3.2) in a control experiment on the small-scale flat sketched in Figure 2. An identification procedure was conducted in [14] to adapt the theoretical model (9) to the measured behavior of the experiment. Due to the large number of parameters influencing the system, we omit most numerical details of this experiment and focus on the method and qualitative comments. More technical details on such experiments can be found in [14].

The inequalities in Theorem 9 define two subsets of the state space that contain the extrema of an interval which is robust controlled invariant. Its lower bound needs to be in $\mathcal{W}=\left\{T \in \mathbb{R}^{4} \mid f(T, \bar{u}, \underline{w}, \underline{\delta}) \succeq_{x} 0\right\}$ and its upper bound in $\mathcal{C}=\left\{T \in \mathbb{R}^{4} \mid f(T, \underline{u}, \bar{w}, \bar{\delta}) \preceq_{x} 0\right\}$. Taking into consideration the forecast on the exogenous temperatures $w$, the chosen robust controlled invariant interval is such that $\overline{T_{f}}=(24,26,26,27)$ and $T_{f}=(21,21,21,21)$. For robust stabilization, we need to find two functions $\underline{T}$ and $\bar{T}$ satisfying Assumption 14 . We choose the first solution presented in Section 4.1: the linear functions between the extrema of the target interval and those of the minimal robust invariant interval $\left(\bar{T}(1)=k_{x}(\bar{u}, \bar{w}, \bar{\delta})\right.$ and $\left.\underline{T}(1)=k_{x}(\underline{u}, \underline{w}, \underline{\delta})\right)$ from Theorem 7 .

The control strategy is implemented with the stabilizing controller described by (5) and (6). When the state reaches the target interval, this controller corresponds to the decentralized affine controller described by (3). The switching scenario of the disturbances is as follows: $t=0 \mathrm{~min}$, lamps 2 and $3 \mathrm{on} ; t=3 \mathrm{~min}$, doors $1-2$ and $2-3$ open; $t=6 \mathrm{~min}$, lamp 4 on, door $3-4$ open; $t=12 \mathrm{~min}$, lamp 3 off, doors $2-3$ and $3-4$ closed; $t=18 \mathrm{~min}$, all lamps off, all doors closed; $t=34 \mathrm{~min}$, all lamps on, all doors open.

The results from this experiment are displayed in Figure 3 . The vertical lines represent the switching times of the discrete disturbances (plain when related to the room, dashed otherwise). The blue curves with circles correspond to the measured temperature in each room. The horizontal red lines are the boundary of the target interval and the dashed red curves are the intervals used in the robust stabilization. If we refer to the notations of the stabilizing controller (6), these red curves correspond to the boundaries $\left(\underline{T}_{i}(\underline{\lambda}(T))\right.$ and $\left.\bar{T}_{i}(\bar{\lambda}(T))\right)$ of the family of robust controlled invariant intervals. The temperatures are measured in Celsius with the left axis. The right axis refers to the controlled ventilation $\left(-u_{i} \in[0,1]\right)$ displayed as the green curve with crosses.

We can see on Figure 3 that the stabilization is achieved 
after 6 minutes: when the stabilization interval coincides with the target interval (in this example, it is the case from the beginning for the lower bound). We can note that a control input saturates only when the state variable of the corresponding room is equal to one of its limits (dashed curves): room 1 at first, then room 3 until the stabilization is achieved. After the stabilization, the controller is able to maintain all state variables in their respective intervals even in the extremal cases of the discrete disturbances (last two steps), which is consistent with the choice of a robust controlled invariant interval.

\section{Conclusion}

In this paper we give a constructive approach of the robust controlled invariance for an interval, which is the ability to control a system to maintain its state in this interval for any value of the disturbances. We then introduce a method to robustly stabilize the system into such an interval for any initial condition. These results apply to a class of monotone systems with local control and are then tested for the temperature control in a smallscale experimental flat equipped with underfloor air distribution. This monotonicity property is not restricted to buildings or temperature control and can be found in many other applications, such as molecular biology, chemical reactions or multi-vehicle systems.

The focus of this paper is on the ability to control (either for invariance or stabilization in an interval) and the results presented above leave a large degree of freedom in the choice of the feedback control strategy to meet the performance specifications of the application: the only requirement on the controller is to use its extrema when the state reaches the boundary of the interval. Therefore the next step of our work, focused on UFAD regulation, is to develop control strategies optimizing comfort and energy consumption while guaranteeing the robustness properties.

\section{References}

[1] A. Abate, A. Tiwari, and S. Sastry. Box invariance in biologically-inspired dynamical systems. Automatica, 45(7):1601-1610, 2009.

[2] D. Angeli and E. D. Sontag. Monotone control systems. IEEE Transactions on Automatic Control, 48(10):1684-1698, 2003.

[3] G. Basile and G. Marro. Controlled and conditioned invariant subspaces in linear system theory. Journal of Optimization Theory and Applications, 3(5):306-315, 1969.

[4] F. S. Bauman and A. Daly. Underfloor air distribution (UFAD) design guide. ASHRAE, 2003.

[5] I. Belgacem and J.-L. Gouzé. Global stability of enzymatic chains of full reversible Michaelis-Menten reactions. Acta biotheoretica, 61(3):425-436, 2013.

[6] F. Blanchini. Set invariance in control. Automatica, 35(11):1747-1767, 1999.
[7] F. Blanchini and S. Miani. Set-theoretic methods in control. Springer, 2007.

[8] L. Chisci and P. Falugi. Asymptotic tracking for constrained monotone systems. IEEE Transactions on Automatic Control, 51(5):873-879, 2006.

[9] R. Ghaemi and D. Del Vecchio. Control for safety specifications of systems with imperfect information on a partial order. IEEE Transactions on Automatic Control, 59(4):982-995, 2014.

[10] M. R. Hafner, D. Cunningham, L. Caminiti, and D. D. Vecchio. Cooperative collision avoidance at intersections: Algorithms and experiments. IEEE Transactions on Intelligent Transportation Systems, 14(3):1162-1175, 2013.

[11] M. D. Lara, L. Doyen, T. Guilbaud, and M.-J. Rochet. Monotonicity properties for the viable control of discrete-time systems. Systems \& Control Letters, 56(4):296-302, 2007.

[12] P.-J. Meyer. Invariance and symbolic control of cooperative systems for temperature regulation in intelligent buildings. PhD thesis, Université Grenoble Alpes, 2015.

[13] P.-J. Meyer, A. Girard, and E. Witrant. Controllability and invariance of monotone systems for robust ventilation automation in buildings. In Proceedings of the $52^{\text {nd }}$ IEEE Conference on Decision and Control, Florence, Italy, 2013.

[14] P.-J. Meyer, H. Nazarpour, A. Girard, and E. Witrant. Experimental implementation of UFAD regulation based on robust controlled invariance. In Proceedings of the $13^{\text {th }}$ European Control Conference, Strasbourg, France, 2014.

[15] H. L. Smith. Monotone dynamical systems: an introduction to the theory of competitive and cooperative systems, volume 41. American Mathematical Soc., 1995.

[16] E. D. Sontag. Monotone and near-monotone biochemical networks. Systems and Synthetic Biology, 1(2):59-87, 2007.

[17] H. L. Trentelman, A. A. Stoorvogel, and M. Hautus. Control theory for linear systems. Springer Verlag, 2001.

[18] E. Witrant, P. Di Marco, P. Park, and C. Briat. Limitations and performances of robust control over WSN: UFAD control in intelligent buildings. IMA Journal of Mathematical Control and Information, 27(4):527-543, 2010.

[19] W. M. Wonham and A. S. Morse. Decoupling and pole assignment in linear multivariable systems: a geometric approach. SIAM Journal on Control, 8(1):1-18, 1970. 